\section{Transient global amnesia: an atypical presentation of acute aortic dissection}

\author{
Monica Masoero, Giannina Leotta, \\ Federico Vischia, Antonio Sechi \\ Emergency Department, San Giovanni \\ Bosco Hospital, Torino, Italy
}

\section{Abstract}

Aortic dissection (AD) is a cardiovascular emergency whose most frequent symptom is chest pain. However, clinical presentation can also be varied and atypical. In the present study we report a case of painless $\mathrm{AD}$, which resulted in a sudden memory loss at presentation. Physical examination and laboratory tests regarding the patient did not show any abnormal findings and a benign transient global amnesia was diagnosed. The day after being discharged, the patient came back to the emergency department because a recurrent episode of severe memory impairment occurred. This time, additional clinical and laboratory signs suggested clinical suspicion of AD. A chest computed tomography angiogram was performed and an extensive $\mathrm{AD}$ type A involving carotid and iliac arteries was found. Our case report shows that acute aortic dissection diagnosis can be difficult if clinical presentation is atypical and must be assessed in the light of various clinical signs and laboratory data.

\section{Introduction}

Aortic dissection (AD) is a cardiovascular emergency whose most frequent symptom is chest pain. However, clinical presentation can be different and atypical. ${ }^{1}$

Not only are neurological symptoms at the onset of aortic dissection frequent (17-40\% of the patients), but they are often dramatic and may conceal the underlying pathological condition. Especially in pain-free dissections (occurring in 5-15\% cases) with predominant neurological symptoms, diagnosis of AD can be difficult and delayed. ${ }^{2,3}$

Despite major advances in diagnostic imaging and progress in therapy, the diagnosis of aortic dissection still remains unsuspected in a high number of patients (up to 28\%), and mortality rate is still high. ${ }^{2,4}$

In literature, only few cases of transient global amnesia (TGA) have been reported in association with acute aortic dissection (AAD) ${ }^{5-16}$

Hence, with the case reported below, we want to emphasize $\mathrm{AD}$ as a rare cause of pure TGA. Indeed, TGA itself often has a benign natural history, but AAD can be life-threatening if it remains undiagnosed.

\section{Case Report}

A 46-year-old man was admitted for the first time to the emergency department (ED) of San Giovanni Bosco Hospital because of an evident, sudden memory loss. His wife witnessed the onset of the episode and reported that since that morning his husband was mentally confused and unable to remember what she had said only few minutes before.

His past medical history was unremarkable. He had a family history of hypertension and diabetes. He denied any known allergy and was not taking any medications at home. At the time of admission the patient was conscious but mentally confused and continously asked questions. He had no other symptoms than headache, chest, neck or abdomen pain. His physical examination was inexpressive and appeared in general good conditions.

The vital parameters showed slight hypotension and the patient complained of a sense of general weakness. There were no signs of trauma or seizure-like activity and the neurological exam was completely normal except for a severe memory impairment.

Electrocardiogram, chest radiography and computed tomography (CT) scan of the head did not reveal any abnormal findings. Few hours after his admission, his ability to form new memories started to improve suggesting a benign pathology. During the period of observation the patient fully recovered; nevertheless, he continued to have difficulties remembering the chronological order of events.

The patient was discharged about after $30 \mathrm{~h}$ from admission with the diagnosis of TGA. However, in order to exclude other differential diagnoses, he was addressed to a further outpatient neurologic evaluation and imaging studies [electroencephalography (EEG), brain magnetic resonance imaging (MRI) and ultrasonographic Doppler examination of carotid and vertebral arteries].

The day after, his wife took him again to the ED because of a recurrent episode of severe memory loss. The patient was conscious, oriented and co-operative, and his neurological examination was still normal with no focal neurological signs.

Upon clinical examination, he presented blood pressure difference between his upper extremities $(128 / 41 \mathrm{mmHg}$ on the left arm $v s$ $98 / 43 \mathrm{mmHg}$ on the right arm), his heart rate was $110 / \mathrm{min}$, regular, and pulse oximetry was $92 \%$.

Chest x-ray and blood gas analysis were nor-
Correspondence: Monica Masoero, Emergency Department, San Giovanni Bosco Hospital, piazza Donatore del Sangue 3, 10124 Torino, Italy.

Tel/Fax: +39.011.4271099.

E-mail: raerte@yahoo.it

Key words: aortic dissection, transient global amnesia, atypical presentation.

Contributions: the authors contributed equally.

Conflict of interests: the authors declare no potential conflict of interests.

Received for publication: 5 April 2013. Revision received: 28 May 2013.

Accepted for publication: 29 May 2013.

This work is licensed under a Creative Commons Attribution 3.0 License (by-nc 3.0).

(C) Copyright M. Masoero et al., 2013 Licensee PAGEPress, Italy

Emergency Care Journal 2013; 9:e11

doi:10.4081/ecj.2013.e11

mal, while electrocardiography (ECG) revealed signs of myocardial ischemia with ST-segment depression in leads V3-V4-V5-V6.

Lab results showed increased levels of cardiac enzymes (Troponine T $0.26 \mathrm{ng} / \mathrm{mL}$ ), elevated white blood cell count $(16,000$ cells $/ \mathrm{mm}$ Neutrophils $84.6 \%)$, stable creatinine (1.0 $\mathrm{mg} / \mathrm{dL}$ ) and marked positive D-dimer (4.93 $\mathrm{mcg} / \mathrm{mL}$ ). Levels of electrolytes were normal, while lactate dehydrogenase (LDH) and $\mathrm{C}$ reactive protein levels were $544 \mathrm{UI} / \mathrm{L}$ and 12.3 $\mathrm{mg} / \mathrm{dL}$, respectively.

Since D-dimer test result was positive, chest CT angiogram was performed and confirmed the suspected aortic disease. A Stanford type A dissection involving the arch and the thoracic aorta up to the abdominal tract and iliac arteries was found. The dissection involved the common and internal carotid artery on the left, sparing the right common carotid artery (Figures 1 and 2).

The patient was immediately transferred to a cardiovascular thoracic surgery facility where an endovascular repairing procedure was successfully performed.

\section{Discussion}

Transient global amnesia is a neuropsychological syndrome in which a sudden, temporary loss of the capacity of creating new memories and a retrograde amnesia of variable intensity occur, whereas consciousness, personal identity and attention are preserved. Patients usually have repetitive questioning and may be confused. ${ }^{5}$ 
Generally, the typical duration of amnesia in TGA is $12 \mathrm{~h}$, and it never lasts more than $24 \mathrm{~h}$. Diagnostic criteria of TGA are purely based on patient's clinical features (Table 1).

Classical AAD has been described as presenting with sudden, severe chest, back, or abdominal pain characterized as ripping or tearing in nature. ${ }^{1}$

Carotid dissection (CD) may represent a threatening complication of $\mathrm{AD}$ or may be an isolate disorder. The disease presents most commonly with headache, Horner's syndrome, stroke, and cranial nerve palsies. ${ }^{7}$

Depending on the extent of the dissection and any involvement of aortic branches, patients with $\mathrm{AD}$ may present with a wide range of symptoms and signs that may be common in other conditions as well, such as ischemic stroke, myocardial infarction or other neurological syndromes including TGA. Our patient had no significant predisposing factors for $\mathrm{AD}$, such as a history of trauma, prior cardiac surgical intervention, or known connective tissue disease, and at the time of admission, none of the most common manifestations of AAD or CD occurred. To our knowledge, literature reports only four other cases of pure TGA syndrome in association to AAD..$^{10,12,13,15}$ In two further cases, focal neurological symptoms were associated (anysocoria, motor deficits) and investigated. ${ }^{8}$ Our patient initially presented with a pure TGA without pain, headache or focal neurological deficits. Still, retrospectively, the sense of general weakness with hypotension, and the slightly prolonged duration of the episode ( $>12 \mathrm{~h}$ ) were atypical of classical TGA. In fact, the patient came back to the ED again with the same amnesic disorder. This time, though, additional clinical and laboratory signs such as difference in blood pressure between the two arms, leukocytosis, elevated D-dimer, $\mathrm{LDH}$ and $\mathrm{C}$ reactive protein

with concomitant increase of cardiac enzymes (Troponine T) and ECG findings of myocardial ischemia aroused the clinical suspicion of $\mathrm{AD}$. Moreover, in benign TGA recurrence is rare as the reported risk of a recurrent attack within 5 years varies between 3 and $20 \%{ }^{3}$ For this patient, thoracic CT scan was performed, because the positive D-dimer test was interpreted together with the clinical features and imaging or laboratory tests mentioned above.

D-dimer measurement is helpful for physicians to order imaging tests. However, our case showed that other important signs should lead to suspect AAD as well as increased D-dimer

Table 1. Transient global amnesia diagnostic criteria.

Attack must be witnessed

Acute onset of anterograde amnesia must be present

No alteration in consciousness must be present

No cognitive impairment other than amnesia must be present

No loss of personal identity must be present

No focal neurology or epileptic features must be present

No recent history of head trauma or seizures must be present

Attack must resolve within $24 \mathrm{~h}$

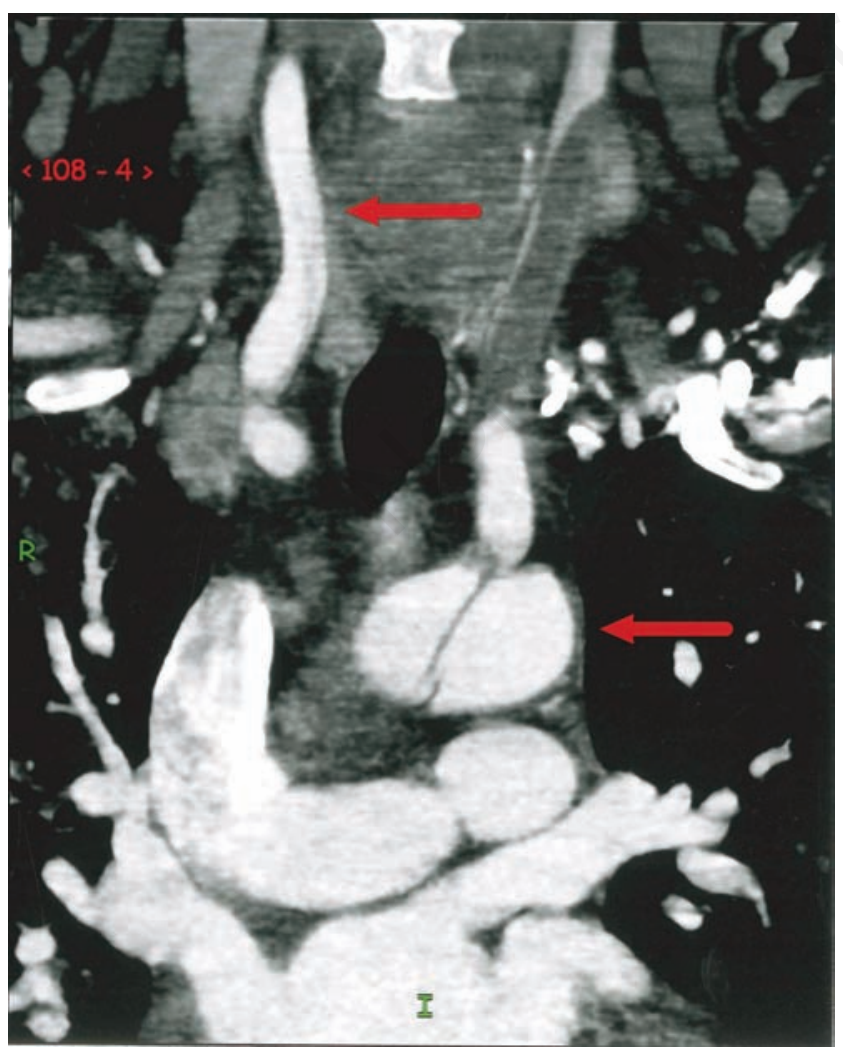

Figure 1. Computed tomography angiography of the dissected aortic arch showing an intimal flap separating the two lumens (right arrow). The dissection involved the left carotid artery (upper-middle arrow).

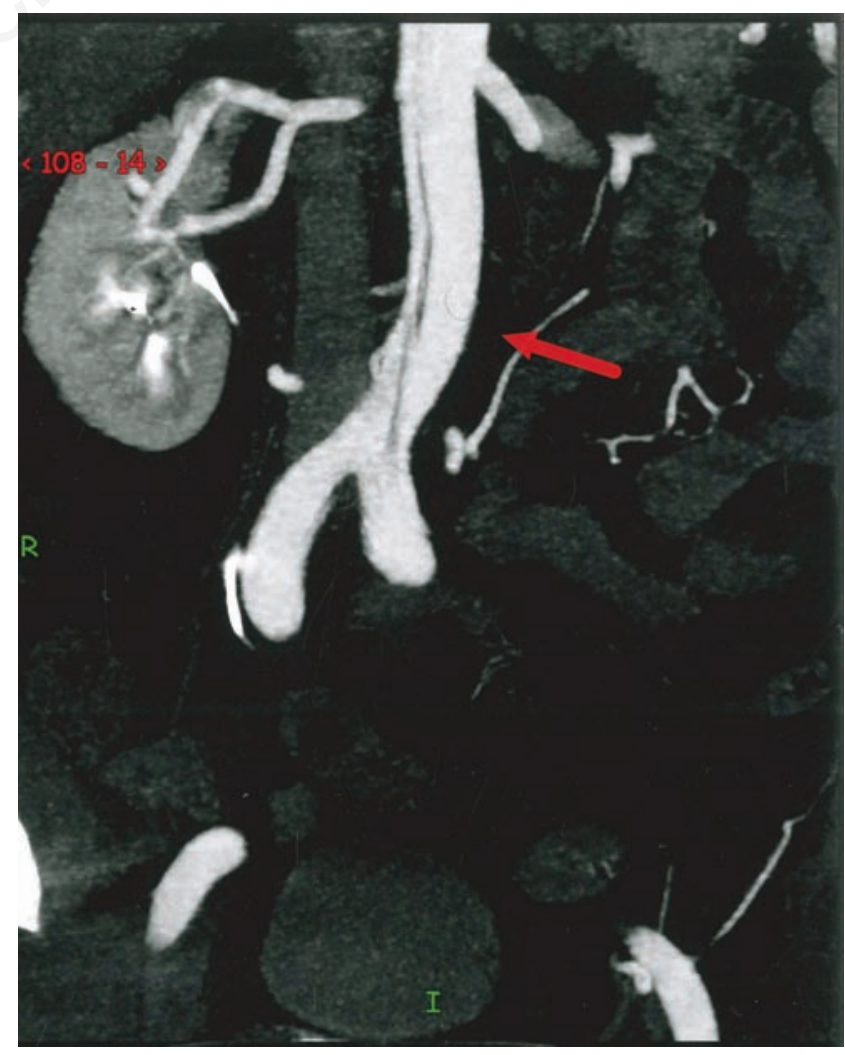

Figure 2. Computed tomography angiography showing the extent of the dissection up to the abdominal tract of the aorta and the iliac arteries (arrow). 
Table 2. Clinical and laboratory signs already described in previously reported cases.

Hypotension/hypertension or difference blood pressure between the arms ${ }^{10,12,13}$

Syncope, paleness ${ }^{12,13,14}$

Chest/back/lumbar pain ${ }^{8,13}$

Fever $^{14}$

Leukocytosis ${ }^{10,14,15}$

Increase of D-dimer levels ${ }^{10}$

Unexplained increase of serum creatinine levels ${ }^{14,15}$

levels. In literature, pulse deficits and difference of blood pressure have been described as initial manifestation of AAD. Even if they are not frequent (from about $10 \%$ to $30 \%$ ), they could suggest the diagnosis of $\mathrm{AD}$ and lead to further diagnostics. In AAD, Troponine T may be increased in up to $18 \%$ of patients at presentation and is reportedly associated with a 3 to-4-fold increased risk of delayed in-hospital diagnosis. ${ }^{9}$

Colotto et al. provided a practical diagnostic algorithm for a new approach to TGA patients admitted to the ED. The first step is to collect information about the onset of amnesic attack. The second step is to carefully check the presence of the clinical and laboratory signs that were described in previously reported cases (Table 2) ${ }^{8,10,12-15}$ If none of the findings in Table 2 is registered, a pure TGA syndrome can be suspected. Otherwise, if any of these abnormalities is present, ED physicians have to suspect the possibility of $A A D$ and urgent angioCT scan of the aorta must be considered..$^{10}$ As previously reported, the first thing to investigate when examining a TGA patient in ED is whether anyone witnesses the onset of amnesia attack: asking relatives, colleagues or any other possible witness is recommended.

Amnesic patient probably cannot recall the onset of symptoms or even pain because the memory of the episode is lost in amnesia. ${ }^{10}$

In literature, sudden paleness, syncope, acute chest or back pain immediately preceding the amnesic disorder have been described as clues of AAD. ${ }^{10,12-14}$

The pathophysiology of TGA remains unknown; there are different hypotheses, but the general consensus is that the amnesic disorder results from transient dysfunction in the hippocampal structures, crucial to memory formation. The dysfunction is often related to idiopathic TGA, but epileptic or vascular origin is also possible. ${ }^{15}$

The relationship between $\mathrm{AAD}$ and TGA is still uncertain. In $\mathrm{AD}$ patients, the propagation of the dissection and false lumen to the cerebral arteries could transiently reduce cerebral blood flow and entail an ischemic stroke with focal signs or a hypoxic encephalopathy with syncope, seizure, or global amnesia. In two case reports, authors postulated that the acute pain of $\mathrm{AD}$ triggered the stress reaction responsible for TGA. ${ }^{12,13}$ In our case there was no pain but neurological signs could also be explained by systemic hypotension and related cerebral perfusion deficit.

Interestingly, neurological findings are frequently evanescent and often fully remitted before admission to the emergency room. ${ }^{3}$ They usually appear at or soon after the onset of dissection. Rapid improvement in such cases is probably the result of only transient arterial occlusion at the moment of propagation of the dissection. ${ }^{2}$

In a recent study, Imamura et al. reported that more than half of the patients with painless $\mathrm{AD}$ were hypotensive. Hypotension occurred in more than $25 \%$ of patients with $\mathrm{AAD}$ and was associated with a higher rate of in-hospital mortality and adverse events. It is possible that hypotension reduces pain because of low wall stress on the aorta. ${ }^{16}$ Also, approximately half of all patients who did not report pain showed neurological symptoms only. ${ }^{3}$

\section{Conclusions}

In conclusion, our case report shows that AAD diagnosis can be difficult and delayed when clinical presentation is atypical. Neurological symptoms are observed in one third of patients with $\mathrm{AD}$ type $\mathrm{A}$ but the absence of the important cardinal symptom pain may misdiagnose AAD. Therefore, ED physicians must have a high index of clinical suspicion for this condition.

Although a pure TGA syndrome in association with $\mathrm{AAD}$ is rare, $\mathrm{AD}$ should be considered in the differential diagnosis of patients who are admitted to the ED with a severe memory impairment and the following clinical and laboratory signs: hypotension/hypertension or difference in blood pressure between the two arms, syncope or paleness, chest, neck or lumbar pain, fever, leukocitosis, and increased Ddimer, myocardial enzymes, LDH or serum creatinine levels.

\section{References}

1. Erbel R, Alfonso F, Boileau C, et al. Diagnosis and management of aortic dissection: recommendations of the task force on aortic dissection, european society of cardiology. Eur Heart J 2001;22:1642-81.

2. Charly G, Wenke D, Frank JE. Neurological symptoms in aortic dissection: a challenge for neurologist. Cerebrovasc Dis 2008; 26:1-8.

3. Gaul C, Dietrich W, Friedrich I, et al. Neurological symptoms in type A aortic dissections. Stroke 2007;38:292-7.

4. Khan IA, Nair CK. Clinical, diagnostic, and management perspectives of aortic dissection. Chest 2002;122:311-28.

5. Owen D, Paranandi B, Sivakumar R, Seevaratnam M. Classical diseases revisited: transient global amnesia. Postgrad Med J 2007;83:236-9.

6. Harrison M, Williams M. The diagnosis and management of transient global amnesia in the emergency department. Emerg Med J 2007;24:444-5.

7. Demiryoguran NS, Karcioglu 0, Topacoglu H, Aksakalli S. Painless aortic dissection with bilateral carotid involvement presenting with vertigo as the chief complaint. Emerg Med J 2006;23:e15.

8. Gaul C, Dietrich W, Tomandl B, et al. Aortic dissection presenting with transient global amnesia-like symptoms. Neurology 2004;63: 2442-3.

9. Rapezzi C, Longhi S, Graziosi M, et al. Risk factors for diagnostic delay in acute aortic dissection. Am J Cardiol 2008;102:1399-406.

10. Colotto M, Maranghi M, Epifania A, et al. Unmasking aortic dissection in patients of transient global amnesia: case report and diagnostic algorithm for the emergency department. BMJ Case Rep 2011;2011:3151.

11. Richard AR, Rosenhecka RE. Transient global amnesia: a case report. Rev Neurol 2008;47:301-3.

12. Bonnet P, Niclot P, Chaussin F, et al. A puzzling case of transient global amnesia. Lancet 2004;364:554.

13. Rosenberg GA. Transient global amnesia with a dissecting aortic aneurysm. Arch Neurol Chicago 1979;36:255.

14. Irioka T, Yamanami A, Yagi Y, et al. Aortic dissection as a possible cause of pure transient global amnesia: a case report and literature review. Neurol Sci 2009;30:255-8.

15. Mondon K, Blechet C, Gochard A, et al. Transient global amnesia caused by painless aortic dissection. Emerg Med J 2007;24:63-4.

16. Imamura $\mathrm{H}$, Sekiguchi $\mathrm{Y}$, Iwashita $\mathrm{T}$, et al. Painless acute aortic dissection diagnostic, prognostic and clinical implications. Circ J 2011;75:59-66. 